\title{
Surface Textured Polymer Fibers for Microfluidics
}

\author{
Adem Yildirim, Muhammad Yunusa, Fahri Emre Ozturk, Mehmet Kanik, \\ and Mehmet Bayindir*
}

This article introduces surface textured polymer fibers as a new platform for the fabrication of affordable microfluidic devices. Fibers are produced tens of meters-long at a time and comprise $\mathbf{2 0}$ continuous and ordered channels (equilateral triangle grooves with side lengths as small as $\mathbf{3 0}$ micrometers) on their surfaces. Extreme anisotropic spreading behavior due to capillary action along the grooves of fibers is observed after surface modification with polydopamine (PDA). These flexible fibers can be fixed on any surface-independent of its material and shape- to form three-dimensional arrays, which spontaneously spread liquid on predefined paths without the need for external pumps or actuators. Surface textured fibers offer high-throughput fabrication of complex open microfluidic channel geometries, which is challenging to achieve using current photolithography-based techniques. Several microfluidic systems are designed and prepared on either planar or 3D surfaces to demonstrate outstanding capability of the fiber arrays in control of fluid flow in both vertical and lateral directions. Surface textured fibers are well suited to the fabrication of flexible, robust, lightweight, and affordable microfluidic devices, which expand the role of microfluidics in a scope of fields including drug discovery, medical diagnostics, and monitoring food and water quality.

are dominantly based on micro-fabrication techniques such as photolithography, laser-writing and plasma etching. ${ }^{[15]}$ Although these techniques have shown to be very successful for shaping material at the microscale, they are impractical for fabrication of affordable microfluidic systems. In addition, fabrication of three-dimensional architectures, which is particularly important for compact and multicomponent microfluidic devices, is challenging with lithography based methods.

Recently, as low-cost competitors of conventional fabrication methods, paper based methods have been developed. ${ }^{[6,16-21]}$ Several research groups demonstrated that, using hydrophobic/hydrophilic patterned paper layers, it is possible to construct low-cost disposable devices that can direct liquid flow in three-dimensions. ${ }^{[22-24]}$ However, fabrication of paper devices include tedious photo-lithography and stacking steps. In addition, produc-

\section{Introduction}

Microfluidic channels that use capillary wicking or directional wetting to control liquid flow are promising alternatives to conventional systems that require external pumps, which limits the simplicity and integrity of the system and thus restricts its use in a number of applications..$^{[-6]}$ In these channels, liquid flow is controlled by anisotropic (i.e. directional) surface structures (e.g. grooves, pillars and nanowires $)^{[7-11]}$ or surface chemistry (e.g. hydrophilic/hydrophobic patterns).$^{[12-14]}$ Such systems are widespread and have been fabricated on a variety of surfaces including glass, silicon and polymers. Current fabrication methods of both physically and chemically patterned surfaces

\footnotetext{
A. Yildirim, M. Yunusa, F. E. Ozturk, M. Kanik, Prof. M. Bayindir

UNAM-National Nanotechnology Research Center and Institute of Materials Science and Nanotechnolog Bilkent University 06800, Ankara, Turkey

E-mail: bayindir@nano.org.tr

Prof. M. Bayindir

Department of Physics

Bilkent University

06800, Ankara, Turkey
}

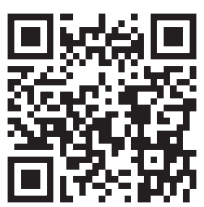

DOI: $10.1002 / \mathrm{adfm} .201400494$ tion of channels with diameters smaller than a millimeter is very challenging due to the rough fibrous structure of the paper.

Here, we utilize fiber drawing for producing polymer fibers with very regular, aligned microscale surface textures. This approach enabled to introduce intrinsic advantages of fibers; covering large areas, flexibility and directionality, to the field of microfluidics. We utilized the fibers as universal macroscopic building blocks to construct three-dimensional open microfluidic devices by simply fixing them to surfaces using double sided tape to form pre-defined architectures. Note that there are examples in the literature that use fiber yarns or fabrics to produce microfluidic channels. ${ }^{[25-28]}$ These studies use bundles of fibers to transport liquids by taking advantage of the porous structure of fiber network in a similar manner to the paper based channels. In this study, on the other hand, we engineered fiber surfaces to produce well-defined micro fluidic channels on individual fibers. We produced on-fiber microfluidic channels in two steps; i) star-shaped very long polymer microfibers are produced by thermal drawing of a surface-structured polyetherimide (PEI) preform, ii) surface of the fibers is coated with polydopamine (PDA) in order to provide them hydrophilic surface chemistry and nano-scale roughness over their textured surface topography. The PDA functionalized star-shaped fibers exhibited extreme anisotropic superhydrophilic behavior, which enables confinement of small liquid portions through microgrooves on the fibers which are tens of centimeters in 
length. Flexibility of the fibers enables production of complex three-dimensional device geometries that can control liquid spreading in both lateral and vertical directions. We first demonstrated preparation of several fiber based microfluidic device components such as open-channels, connections, bridges and switches on both planar and unconventional geometries. We then developed a proof of principle colorimetric protein assay for human serum albumin (HSA) detection.

\section{Results and Discussion}

\subsection{Fabrication of Textured Fibers}

Thermal fiber drawing is a well-established top-down fabrication method which is used for production of various functional micro and nano structured fibers. ${ }^{[29-32]}$ This simple and robust manufacturing process enables to maintain well defined material geometries along the length of the fiber. In this work, we utilized fiber drawing method to modify fiber surface morphology in order to obtain enhanced anisotropic wetting on fiber surfaces. This is the first application of fiber drawing technique for modifying surface morphology of fibers to the extent of our knowledge. Surface textured fibers were fabricated in two main steps. In the first step, a PEI preform was shaped in lathe to obtain a macroscopic star-shape. PEI was chosen for its ability to preserve its initial shape particularly well during fiber drawing. ${ }^{[31]}$ Star-shaped preform was drawn under suitable mechanical stress and thermal parameters to obtain fibers of several meters in length and few hundred microns in diameters (Figure 1A). Precise control of diameter over a wide range is possible at this stage by simply changing draw parameters. Figure 1B-i shows the photograph of star-shaped preform after thermal drawing process, where conservation of the star-shape during size reduction can be clearly seen. Figure 1B-ii shows the photograph of several meter long flexible star-shaped fiber
A

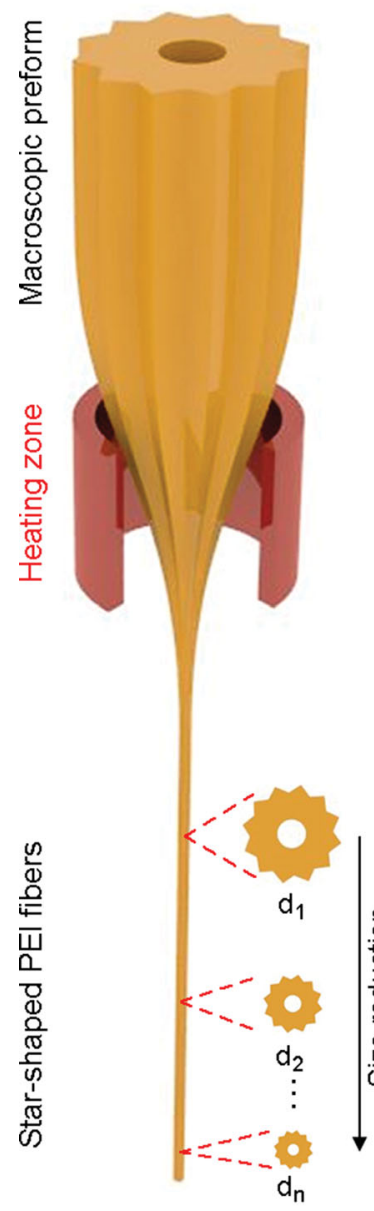

B
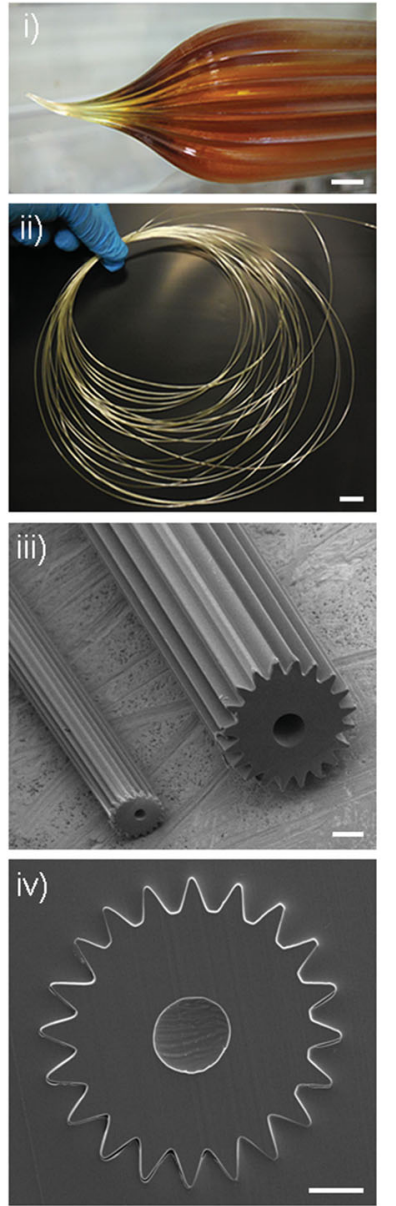

C

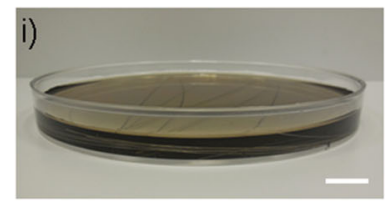

ii)

Uncoated

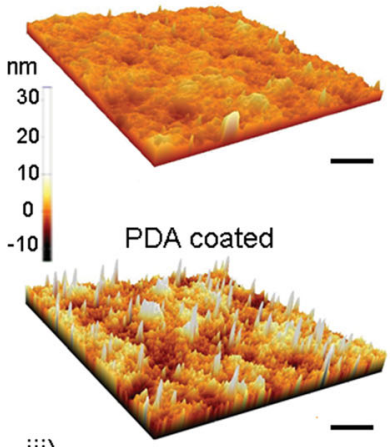

iii)

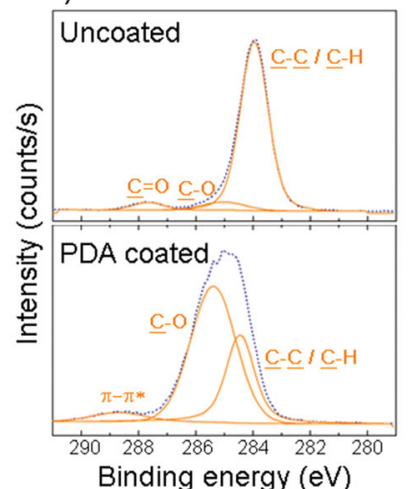

Figure 1. Fabrication of star-shaped PEI fibers. (A) Textured polymer microfibers were fabricated by thermal drawing from a macroscale preform. (B) (i) Photograph of preform after thermal drawing (scale bar: $5 \mathrm{~mm}$ ). (ii) Photograph of drawn microstructured fibers of several meters showing their robustness and flexibility (scale bar: $1 \mathrm{~cm}$ ). (iii) SEM micrograph of a $500 \mu \mathrm{m}$ diameter and a $200 \mu \mathrm{m}$ diameter textured polymer fiber showing microscale ordered grooves on their surfaces (scale bar: $100 \mu \mathrm{m}$ ). (iv) SEM cross section of ordered microgrooves on textured fibers. Fibers preserve their 20-point star-shape along their length (scale bar: $50 \mu \mathrm{m}$ ). (C) PDA coating and characterization. (i) PDA coating is achieved by dipping the fibers into dopamine solution for a determined time (scale bar: $1 \mathrm{~cm}$ ). (ii) AFM micrographs of uncoated and PDA coated fiber surfaces. After surface modification nanoscale roughness is introduced with PDA coating to the fiber surface (scale bar: $1 \mu \mathrm{m}$ ). (iii) Cls XPS spectra of uncoated and PDA coated fibers. 
bundle. The surface texture of the fibers was investigated using SEM (Figure 1B-iii, iv) which showed 20 continuous, perfectly aligned grooves on the fibers. We observed that even after 150 fold size reduction from $3 \mathrm{~cm}$ (diameter of the preform) to $200 \mu \mathrm{m}$ (thinnest fiber produced in this study), star-shape with a groove width of only 30 microns, is preserved. We also prepared fibers with smooth surfaces using a non-structured preform in order to investigate the effect of surface texture on wetting properties of the fibers (Supporting Information, Figure S1). In the second step, fibers were coated with PDA by dipping into dopamine solution in order to make the hydrophobic fiber surface hydrophilic (Figure 1C-i). ${ }^{[33]}$ Surface topography images of uncoated and PDA coated smooth fibers indicate formation of PDA nanoparticles after surface modification (Figure 1C-ii).

The chemical structure of the formed polymer (i.e. melanin like polymer synthesized using dopamine, commonly referred as polydopamine $(\mathrm{PDA}))^{[34]}$ on the surface of the fibers was investigated using XPS, FT-IR and UV-Vis absorption spectroscopies. Figure 1C-iii shows the carbon 1s XPS spectra of PDA coated and uncoated PEI fibers. For uncoated fibers, very weak C-O peak was observed. After PDA modification this peak becomes much more intense which suggests the presence of PDA on the surface which is in accordance with previous works. ${ }^{[35,36]}$ UV-Vis absorption spectra of the $24 \mathrm{~h}$ polymerized DA solution shows the typical broad band absorption of PDA (Supporting Information, Figure S2a). ${ }^{[37]}$ The transmission of PEI film significantly reduced after coating with PDA (24 h) due to the broad band light absorption of PDA (Supporting Information, Figure S2b). FT-IR spectrum of PDA ( $24 \mathrm{~h}$ polymerized) powder shows absorption bands of indoline, indole, carbonyl, amino and hydroxyl groups of PDA which is also in accordance with previous reports (see Supporting Information for more information, Figure S3 and Table S1). ${ }^{[36,37]}$ Note that exact chemical structure (it is assumed that PDA is composed of covalently linked dihydroxyindole, indoledione, and dopamine units) or microstructure (e.g. linear polymers, cylclic oligomers, physical aggregates or combinations of these structures) of PDA is still not fully resolved; ${ }^{[38]}$ therefore we cannot conclusively determine the exact structure of the synthesized polymer in our experimental conditions. Nevertheless, our chemical analysis indicates a PDA polymer deposited onto the fiber surfaces.

\subsection{Extreme Anisotropic Wetting on Free-standing Textured Fibers}

Single free-standing PDA coated star-shaped fiber can transport water along the micron sized channels to its entire length in a few seconds when a water droplet was introduced from the middle of the fiber (Figure 2A-i). This extreme directional wetting property was not observed on PDA coated smooth fibers or on uncoated star-shaped fibers (Figure S4, Supporting Information) which indicates that the three scale roughness and hydrophilic surface chemistry is essential to provide total wetting of the fiber surface. Figure 2B-ii and iii show the edge of the fiber with a diameter of $500 \mu \mathrm{m}$ whose surface grooves were filled with blue-colored water. In addition, we observed that microgrooves transport the water along the fiber but not to their neighbor grooves (Figure 2B-iv). Therefore, it is possible to select liquid transport channels by adjusting the droplet size and contact area.

In order to better understand the extreme anisotropic wetting behavior of the grooved fiber surface, we applied spontaneous capillary flow equation (Supporting Information, Equation S1). ${ }^{[3]}$ PEI is a hydrophobic material with a water contact angle of $97^{\circ}$ and after modification with PDA, surface becomes hydrophilic with a water contact angle of $32^{\circ}$ (Supporting Information, Figure S5). Equation S1 yields contact angle limit of about $60^{\circ}$ for spontaneous spreading on our grooved geometry (see Supporting Information for details). Therefore capillary action is favorable in PDA coated star-shaped fibers but not in uncoated ones which supports our experimental observations.

\subsection{Preparation of Fiber Arrays}

After demonstrating the extreme wettability on free-standing fibers, we prepared several fiber arrays by fixing them to paperboards using a double sided adhesive tape and characterized their wetting properties (Figure 2B). We measured the water contact angles of the surfaces in both parallel (i.e. contact line is parallel to the fiber orientation) and perpendicular (i.e. perpendicular to the fiber orientation) directions (Figure 2C). Uncoated arrays of both smooth and star-shaped fibers demonstrated improved hydrophobicity compared to the PEI film due to the single or double micro scale roughness of these surfaces. On the other hand, after PDA functionalization, fibers become very hydrophilic. However, the effect is exceptional in the case of PDA coated star-shaped fiber array; contact angle values reach $0^{\circ}$ in both directions. The extreme anisotropic superhydrophilic behavior of the textured fiber array was also visualized in Figure 2D. It is important to note that, $8 \mu \mathrm{L}$ of dyed water droplets spread to the $7 \mathrm{~cm}$ long fiber array within only 5 seconds (Supporting Information, Video S1). Also, similar to the on-fiber micro channels, these macroscopic channels transport the liquid along the fiber but not to the neighbor fibers which avoids intermixing. In addition, we investigated the spreading rate of water on $6 \mathrm{~cm}$ long fiber arrays consisting of four fibers, with each array consisting of different sized fibers (200 $\mu \mathrm{m}, 300 \mu \mathrm{m}$ and $500 \mu \mathrm{m}$ ). We observed that filling time decrease with increasing fiber size. Further, spreading distance $(L)$ is accurately proportional to square root of time $(\sqrt{t})$, which is in accordance with previous reports that investigate spreading dynamics on v-shaped grooves ${ }^{[39-41]}$ (see Supporting Information for more information, Section S5 and Figure S6).

\subsection{Three-dimensional Microfluidic Channels}

Open microfluidic channels that can control liquid flow in both lateral and vertical directions can be easily prepared using PDA coated star-shaped fibers owing to their flexibility. For instance, Figure 3A shows a curved $15 \mathrm{~cm}$ long open microfluidic channel which consists of five $300 \mu \mathrm{m}$ sized fibers that successfully direct the flow of $40 \mu \mathrm{L}$ of red colored water in its predefined path. Water filled the channel approximately in 2.5 minutes without any external influence. The observed 


\section{A}
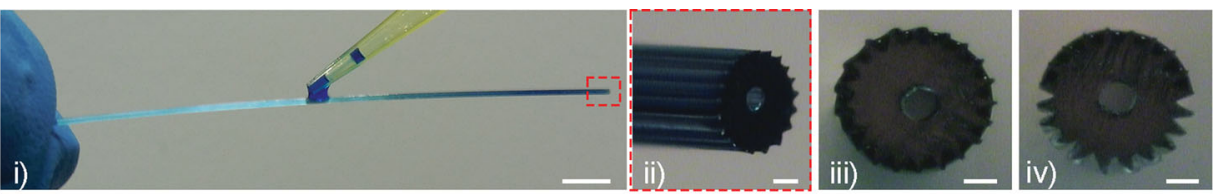

$\mathrm{B}$
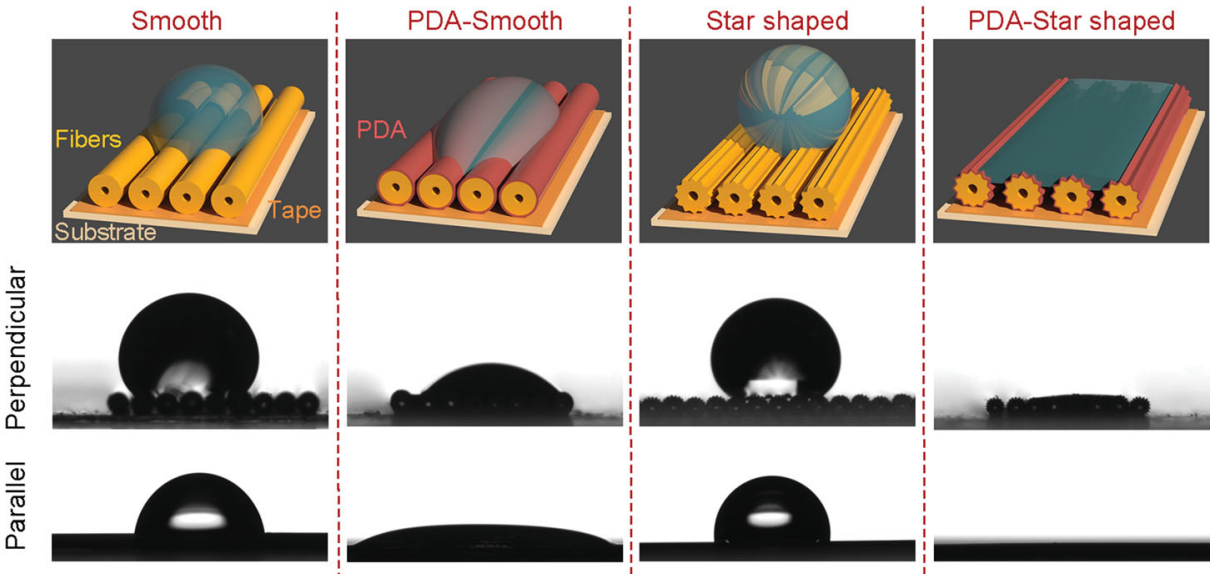

C
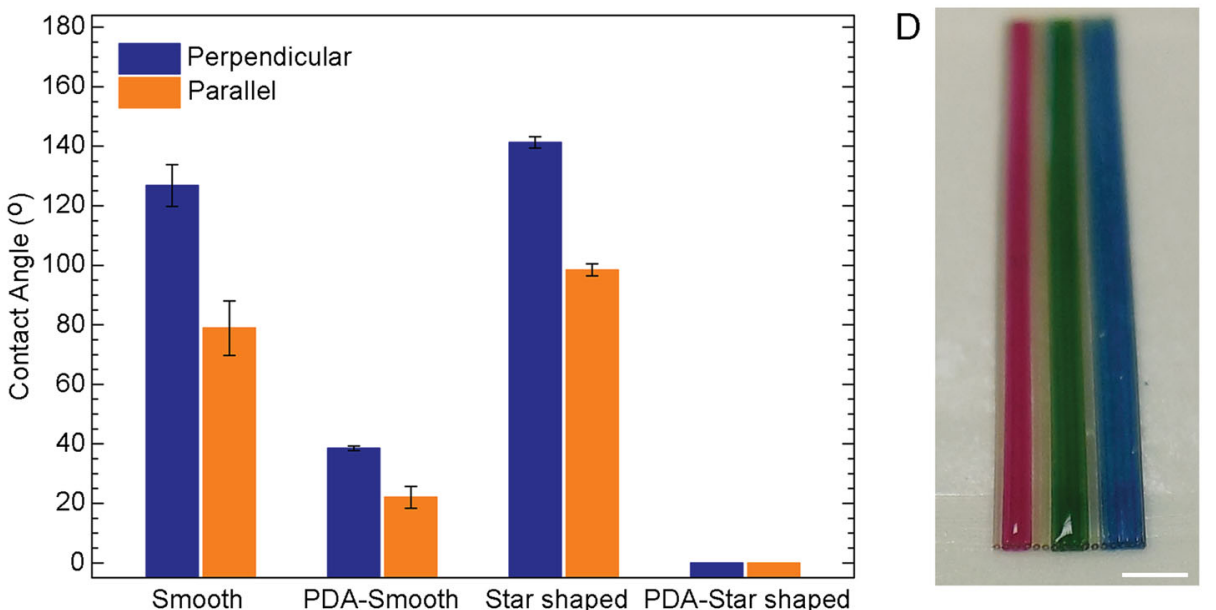

Figure 2. Exceptional anisotropic wetting of star-shaped fibers. (A) Dyed water is introduced to a free standing PDA coated star-shaped fiber. (i) Each fiber has 20 individual, parallel micro-channels on its surface (scale bar: $3 \mathrm{~mm}$ ). (ii) Close up photograph of the fiber end (scale bar: $100 \mu \mathrm{m}$ ). (iii) Cross sectional view of the dyed water introduced fiber (scale bar: $100 \mu \mathrm{m}$ ). (iv) The case when the water is introduced from the upper surface. Only the channels that come into contact with the water are filled (scale bar: $100 \mu \mathrm{m}$ ). (B) Wetting behavior of fiber arrays. Fiber surfaces are prepared by simply aligning the fibers on an adhesive tape. Parallel and perpendicular corresponds to the direction contact angle measurement is taken. When there is no PDA coating, star-shaped fibers are more hydrophobic from smooth fibers due to increased roughness of the PEI surface. After PDA modification of the surfaces, both smooth and star-shaped fibers show increased hydrophilic behavior. However, water is spread to the whole length of the fiber for star-shaped fibers. (C) Contact angle measurements of fiber array surfaces. (D) A fiber array comprised of 16 fibers of length $6 \mathrm{~cm}$. Dyed water of different colors is introduced to the surface (scale bar: $2 \mathrm{~mm}$ ).

longer filling time for this curved channel, compared to $7 \mathrm{~cm}$ long fiber array mentioned above (Video S1) is due to its higher fiber length and curved shape (see Supporting Information for more information, Section S6, Figure S7 and Video S2).

Controlling liquid spreading from a reservoir to several channels or one channel to another is needed to construct multichannel microfluidic devices. Figure 3B shows distribution of water from a reservoir to eight different channels which are composed of single $500 \mu \mathrm{m}$ sized fibers. Also, we demonstrated that end to end added channels can transmit water from one to another (Supporting Information, Figure S8). In addition, we showed that using PDA-star shaped fibers, it is possible to design connection architectures. For example, when water is introduced from one end of the upper channel of two cross over channels, top channel transfers some of the water to the bottom channel (Figure 3C). The close up image of this connection (Figure 3D) demonstrates the water transfer between close contact stacked channels (Supporting Information, Video S3). Introducing a $200 \mu \mathrm{m}$ gap between top and bottom channels with the addition of spacers (uncoated $500 \mu \mathrm{m}$ fibers) prevented the mixing of upper and lower liquid flows (Figure 3F). This enables us to construct 'bridge' architectures that are capable of transporting liquid streams crossover another. For instance, Figure 3E demonstrates a three-dimensional array composing 

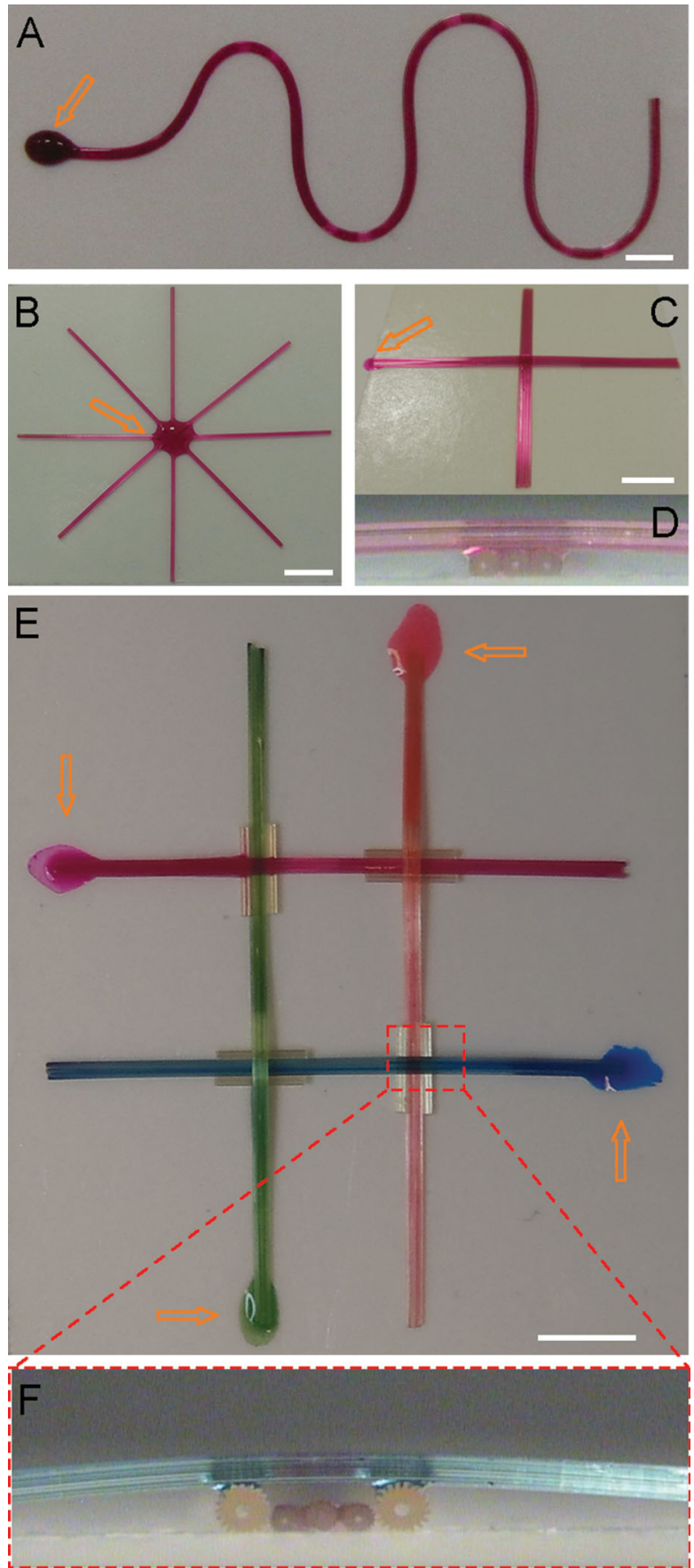

Figure 3. Construction of microfluidic networks with star-shaped fibers. (A) Spontaneous wetting is achieved in about 2.5 minutes to the whole length of $15 \mathrm{~cm}$ fibers aligned in a curved path (scale bar: $1 \mathrm{~cm}$ ). Orange arrows show the point of dyed water introduction. (B) Distribution of liquid to multiple channels from single drop. Single fibers of diameter $500 \mu \mathrm{m}$ spread the dyed water to any direction (scale bar: $5 \mathrm{~mm}$ ). (C) Connection of microfluidic channels by stacking fiber arrays. Liquid spread on the above array is transferred to the below array (scale bar: $5 \mathrm{~mm}$ ). (D) Shows a close up view of the connection point. (E) A three-dimensional system comprising of 4 channels crossing one another. At the intersection points, two uncoated larger hydrophobic star-shaped fibers are placed at the both sides of the below channel serve as spacers to prevent contact between below and above channels (bridge geometry). (scale bar: $5 \mathrm{~mm}$ ). (F) Shows close-up view of the bridge from a different angle. of 4 channels that cross one another. None of the liquid streams intermixed in all of the 4 bridges.

\subsection{Switch Design}

Switch architectures give the user ability of on-demand liquid transport; i.e. which and when the channels should be activated. ${ }^{[19]}$ Here, we designed two different switch geometries (Figure 4). The first switch geometry is the same with the bridge geometry discussed above. We simply pressed the bridge using a sharp tip (tweezer) and provided contact between top and bottom channels, which results in immediate filling of the below channel (Figure 4A). In the second design, we constructed free-standing channels just a few millimeters above the fixed bottom channels (Figure 4B). We first fill the freestanding channels and then activate the below channels one at a time by simply bending the free-standing channels and providing a contact between top and bottom channels. All of the three switches operated successfully.

\subsection{Flexibility of the Channels}

Flexibility of the PEI fibers enables us to construct microfluidic channels on complex three-dimensional objects. Figure 5A shows the $15 \mathrm{~cm}$ long rolled microfluidic channel (comprising of four $200 \mu \mathrm{m}$ thick PDA-star shaped fibers) around a glass rod. The array can transport $50 \mu \mathrm{L}$ of colored water along the channel approximately in 6 minutes. This example demonstrates that surface textured fiber based open microfluidic channels can also operate against gravitational force. Another example of a microfluidic channel demonstration on a threedimensional object was given in Figure S9 (Supporting Information) which shows liquid climbing a ramp. Furthermore, microfluidic channels can be prepared on flexible substrates which can operate under bending. For instance, Figure 5B shows three bended microfluidic channels, prepared on a polycarbonate film, which are filled with differently colored water portions.

\subsection{Protein Assay}

As a proof of concept demonstration that our method is suitable to fabricate disposable and low-cost medical test kits, we prepared a protein assay for detection of human serum albumin (HSA) which is the most abundant protein in human blood. The assay is based on the color change of the bromophenol blue (BPB) dye in the presence of HSA. ${ }^{[42,43]}$ We used filter paper as detection zones, where it is possible to observe the color change upon protein addition, as paper is an intrinsically hydrophilic and macroporous material that can absorb definite volume of water depending on its size. ${ }^{[24]}$ In a simple experiment, we demonstrated that paper in contact with the fiber microfluidic channels can easily wick the liquid on the channels (Figure 6A).

In the first colorimetric assay (Figure 6B), we constructed a microfluidic channel (composing of six $300 \mu \mathrm{m}$ diameter 

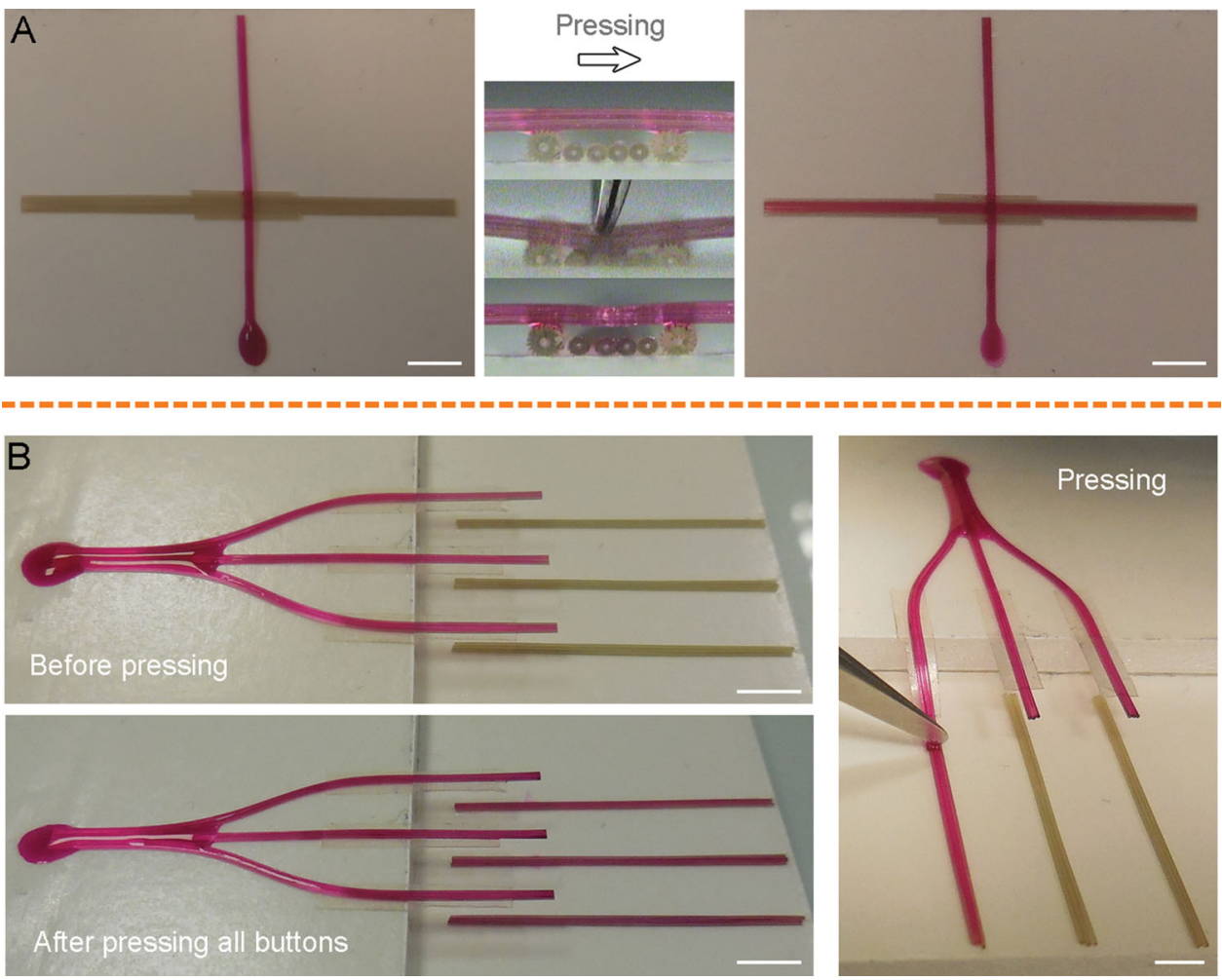

Figure 4. Switches that control water spreading. Utilizing the observed liquid transfer upon stacked fibers, a simple button mechanism is shown. (A) Initially there is no liquid at the below array, there is no intermixing between above and below arrays (i) (scale bar: $5 \mathrm{~mm}$ ). (ii) When the above array is pressed down with a tweezer it comes into contact with the below array and spontaneous spreading begins at the below array instantaneously. (iii) After release of the above array it relaxes back to its initial position and there is no further liquid transfer between channels. At the end stage water is distributed to the whole length of the below array. (B) Interplanar button mechanism. This example shows a button for liquid transfer between planes. Spontaneous spreading begins instantaneously by contact with the already wet array (scale bar: $5 \mathrm{~mm}$ ).

fibers) which is then divided into three sub-channels and connected to circular filter paper pieces $(6 \mathrm{~mm}$ in diameter) at the end of each channel. Then, $2 \mu \mathrm{L}$ of protein assay reagents $\left(0.1 \mathrm{mg} \mathrm{mL}^{-1} \mathrm{BPB}\right.$ in glycine buffer $(10 \mathrm{mM})$ at $\left.\mathrm{pH} 2.3\right)$ were dropped onto the paper detection zones and dried under ambient air. After addition of $20 \mu \mathrm{L}$ of HSA solution $(5 \mathrm{mg}$ $\mathrm{mL}^{-1}$ in PBS, $\mathrm{pH}$ 7.4) to the main microfluidic channel, color of the detection zones changed from light yellow to blue, within a minute. In addition, a quantitative HSA analysis was demonstrated (Supporting Information, Figure S10) using the assay. We observed that assay can easily identify HSA concentrations of between 0.2 and $5 \mathrm{mg} \mathrm{mL}^{-1}$.

After this simple demonstration, we designed a more complex protein assay which consists of 1 BPB channel, 3 protein channels, 3 switches, 3 detection zones and 3 control zones (Figure 6C). In this design, one can select the channel on which the experiment will be performed. It is important to note that, after activating the switches, both control and detection zones are filled with BPB solution; on the other hand protein solution only fills the detection zones. Initially, the BPB channel was filled with $50 \mu \mathrm{L}$ of BPB solution. Then, we added $4 \mu \mathrm{L}$ of HSA solution $\left(10 \mathrm{mg} \mathrm{mL}^{-1}\right)$ to the first channel and activate the first switch. Immediately after pushing the switch we observed a blue color in detection zone (color of BPB in the presence of HSA) and light yellow color in the control zone. Note that, protein solution only fills the detection zones because there is a gap on the fiber channel under the control spots (Supporting Information, Figure S11). Although the two channels are connected over the gap with a piece of paper, protein solution was not transferred to the control channel since this small amount of $(4 \mu \mathrm{L})$ protein solution is completely absorbed by the paper. We repeated this procedure for the other two channels and observed that all channels worked properly; the color changes of analysis and control spots are clearly distinguishable (Supporting Information, Table S2).

\section{Conclusions}

We demonstrated that PDA functionalized surface textured polymer fibers can be utilized as universal building blocks to produce microfluidic devices that are; i) not restricted to conventional planar geometries, ii) mechanically flexible and robust, and iii) easily adaptable for new designs. Each of the 20 microchannels on the star-shaped fibers was spontaneously filled with water upon contact due to capillary action, which enables free-standing microfluidics on the fibers. Several microfluidic device components including channels, connections, bridges and switches were designed by arrays of star-shaped fibers, which enable three-dimensional and programmable control over liquid flow. Furthermore, we fabricated two diagnostic devices which can give colorimetric response in 

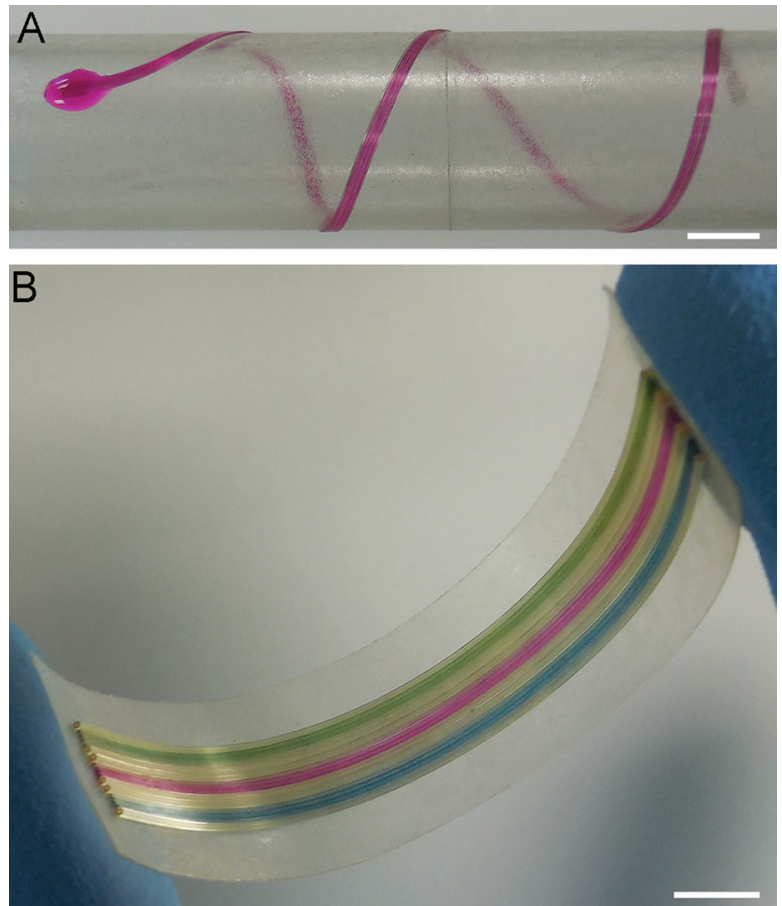

Figure 5. Fiber based microfluidics unrestricted with planar based systems. Since fibers are flexible, the observed anisotropic wetting behavior can be achieved on any surface. (A) Spontaneous wetting on fibers of length $15 \mathrm{~cm}$ rolled around a glass tube (scale bar: $5 \mathrm{~mm}$ ). (B) Microfluidic channel array on a flexible surface. The array consists of about 120 microchannels on 12 fibers, uncoated $500 \mu \mathrm{m}$ star-shaped fibers are added in between the coated $300 \mu \mathrm{m}$ fibers for avoiding the delivery of liquid to the wrong array (scale bar: $5 \mathrm{~mm}$ ). the presence of HSA. We believe that this high throughput, low cost and simple method can be used in designing disposable microfluidic devices for early stage diagnosis and point-of-care analysis, as well as proving to be a novel fabrication scheme for further possibilities that require tuning of liquid behavior on large-areas and flexible systems.

\section{Experimental Section}

Preform Preparation: First, PEI films (previously cleaned and kept in vacuum at $120^{\circ} \mathrm{C}$ for a day) are tightly rolled around a Teflon rod under a clean pressure flow hood until the diameter reaches $30 \mathrm{~mm}$. Then, in order to fuse PEI layers, the preform is thermally consolidated under vacuum $\left(8 \times 10^{-3}\right.$ torr $)$ in two stages. In the first stage, preform is heated to $180^{\circ} \mathrm{C}$ at a rate of $15^{\circ} \mathrm{C} \mathrm{min}^{-1}$ and kept at this temperature for $4 \mathrm{~h}$. In the second stage, the temperature is increased to $257^{\circ} \mathrm{C}$ at a rate of $2{ }^{\circ} \mathrm{C} \mathrm{min}^{-1}$ and preform is consolidated at this temperature for 45 min. Finally, consolidated preform was shaped in lathe to give the final 20 pointed star-shape.

Thermal Fiber Drawing: After obtaining triangular grooves on the polymer preform, macroscopic star-shaped structure was extended to a length of tens of meters, and scaled down to diameters at the micro scale via thermal fiber drawing. The surface textured preform was vertically fed in to a furnace with a speed of $8 \mathrm{~mm} / \mathrm{min}$. The furnace was heated to approximately $305{ }^{\circ} \mathrm{C}$, and an adjustable load was applied to preform with a constant speed motor. Under these optimized parameters, polymer micro fibers that maintain the initial star-shape are drawn. Fiber diameter is controlled by precise tuning of load; diameter is scaled down to desired values as small as $200 \mu \mathrm{m}$ by increasing capstan speed.

PDA Coating: Fibers were coated with PDA according to a previous report. ${ }^{[33]}$ Briefly, $120 \mathrm{mg}$ of tris(hydroxymethyl)aminomethane (SigmaAldrich, USA) is dissolved in $100 \mathrm{~mL}$ of water and $\mathrm{pH}$ of the solution is adjusted to 8.5 using dilute hydrochloric acid. Then $10 \mathrm{mg}$ of dopamine
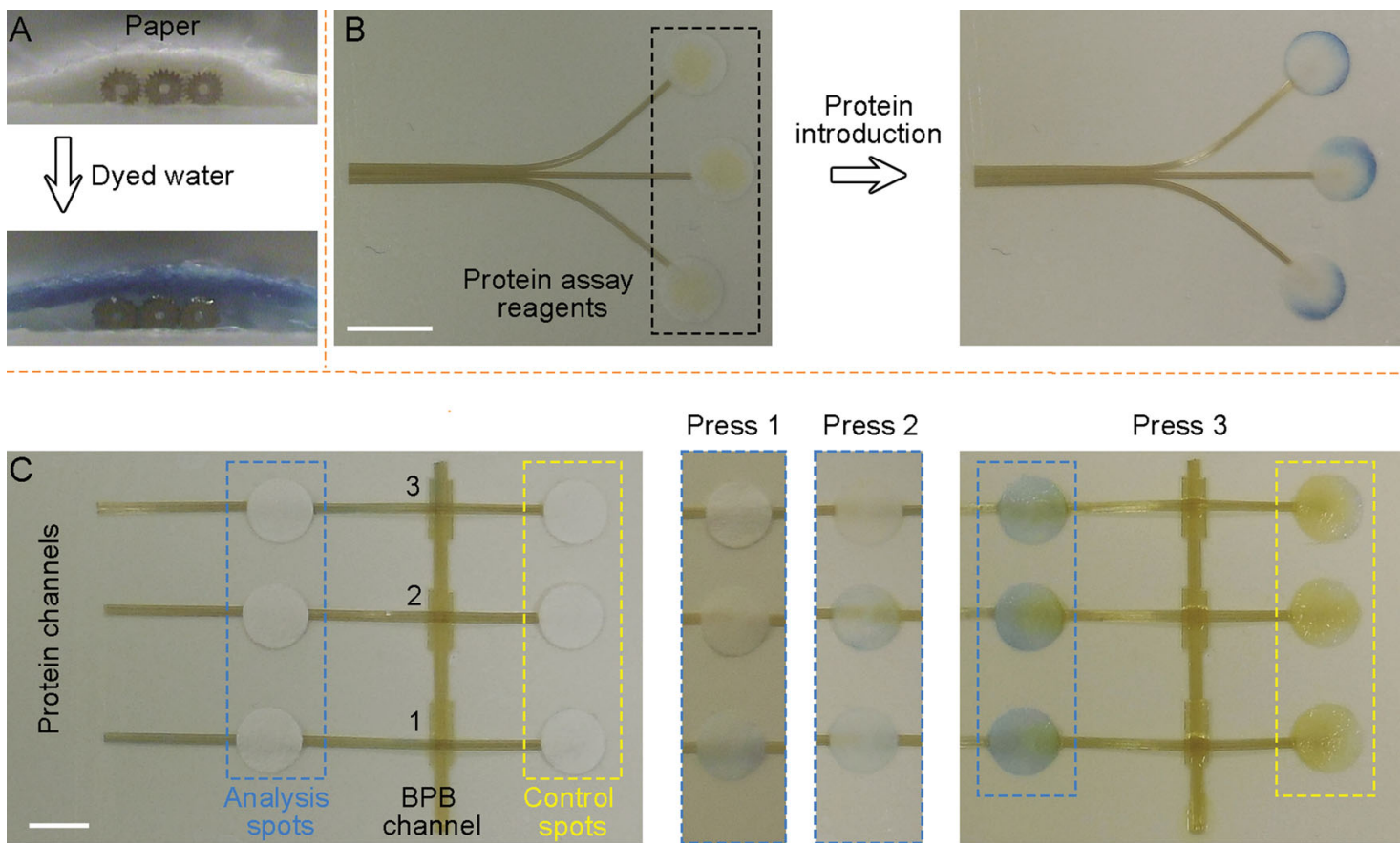

Figure 6. Protein assays. (A) Demonstration of water transfer from a microfluidic channel to a piece of filter paper. (B) A protein assay composing of three detection zones. Upon addition of HSA immediate color change occurred at all detection zones (scale bar: $1 \mathrm{~cm}$ ). (C) A protein assay composing of three analysis and control spots and three switches. After activation of all switches color change was observed in analysis spots (scale bar: $5 \mathrm{~mm}$ ). 
(Sigma-Aldrich, USA) is added to this solution. Immediately after dopamine addition, approximately $15 \mathrm{~cm}$ cleaned (sonicated in IPA for 3 minutes) smooth or star-shaped fiber pieces are dipped into this solution for 1 to $24 \mathrm{~h}$. The color of the solution turns to dark brown from transparent over time indicating the PDA formation.

\section{Supporting Information}

Supporting Information is available from the Wiley Online Library or from the author.

\section{Acknowledgements}

We thank Prof. Çağlar Elbüken and Urandelger Tuvshindorj for fruitful discussions, A. Eren Öztürk for his helps in modelling of 3D figures, and Murat Dere for his helps on preform preparation and fiber drawing. This work is supported by TUBITAK under the Project No. $110 \mathrm{M} 412$. The research leading to these results has received funding from the European Research Council under the European Union's Seventh Framework Programme (FP/2007-2013) / ERC Grant Agreement $n$. 307357. M. B. acknowledges partial support from the Turkish Academy of Sciences (TUBA).

Received: February 12, 2014 Revised: March 8, 2014 Published online: April 17, 2014

[1] J. Atencia, D. J. Beebe, Nature 2005, 437, 648.

[2] H. Gau, S. Herminghaus, P. Lenz, R. Lipowsky, Science 1999, 283, 46.

[3] B. P. Casavanta, E. Berthier, A. B. Theberge, J. Berthier, S. I. Montanez-Sauri, L. L. Bischel, K. Brakke, C. J. Hedman, W. Bushman, N. P. Keller, D. J. Beebe, Proc. Natl. Acad. Sci. USA 2013, 110, 10111.

[4] T. A. Duncombe, E. Y. Erdem, A. Shastry, R. Baskaran, K. F. Böhringer, Adv. Mater. 2012, 24, 1545.

[5] S. H. Lee, A. J. Heinz, S. Shin, Y. Jung, S. Choi, W. Park, J. Roe, S. Kwon, Anal. Chem. 2010, 82, 2900.

[6] M. Hitzbleck, R. D. Lovchik, E. Delamarche, Adv. Mater. 2013, 25, 2672.

[7] R. Seemann, M. Brinkmann, E. J. Kramer, F. F. Lange, R. Lipowsky, Proc. Natl. Acad. Sci. USA 2005, 102, 1848.

[8] V. Liimatainen, V. Sariola, Q. Zhou, Adv. Mater. 2013, 25, 2275.

[9] D. Xia, S. R. J. Brueck, Nano Lett. 2008, 8, 2819.

[10] J. Y. Chung, J. P. Youngblood, C. M. Stafford, Soft Matter 2007, 3, 1163.

[11] V. Jokinen, M. Leinikka, S. Franssila, Adv. Mater. 2009, 21, 4835.

[12] B. Zhao, J. S. Moore, D. J. Beebe, Science 2001, 291, 1023.

[13] K. Tsougeni, D. Papageorgiou, A. Tserepi, E. Gogolides, Lab Chip 2010, 10, 462.
[14] N. M. Oliveira, A. I. Neto, W. Song, J. F. Mano, Appl. Phys. Express 2010, 3, 085205

[15] D. Xia, L. M. Johnson, G. P. López, Adv. Mater. 2012, 24, 1287.

[16] A. W. Martinez, S. T. Phillips, M. J. Butte, G. M. Whitesides, Angew. Chem. Int. Ed. 2007, 46, 1318.

[17] A. W. Martinez, S. T. Phillips, E. Carrilho, S. W. ThomasIII, H. Sindi, G. M. Whitesides, Anal. Chem. 2008, 80, 3699.

[18] Y. Lu, W. Shi, L. Jiang, J. Qin, B. Lin, Electrophoresis 2009, 30, 1497.

[19] A. W. Martinez, S. T. Phillips, Z. Nie, C. Cheng, E. Carrilho, B. J. Wiley, G. M. Whitesides, Lab Chip 2010, 10, 2499.

[20] X. Li, J. Tian, G. Garnier, W. Shen, Colloids Surf. B: Biointerfaces 2010, 76, 564

[21] H. Liu, R. M. Crooks, J. Am. Chem. Soc. 2011, 133, 17564.

[22] A. W. Martinez, S. T. Phillips, G. M. Whitesides, Proc. Natl. Acad. Sci. USA 2008, 105, 19606.

[23] G. G. Lewis, M. J. DiTucci, S. T. Phillips, Angew. Chem. Int. Ed. 2012, $51,12707$.

[24] S. T. Phillips, G. G. Lewis, MRS Bull. 2013, 38, 315.

[25] X. Li, J. Tian, W. Shen, ACS Appl. Mater. Interfaces 2010, 2, 1.

[26] F. Vatansever, R. Burtovyy, B. Zdyrko, K. Ramaratnam, T. Andrukh, S. Minko, J. R. Owens, K. G. Kornev, I. Luzinov, ACS Appl. Mater. Interfaces 2012, 4, 4541.

[27] P. Bhandari, T. Narahari, D. Dendukuri, Lab Chip 2011, 11, 2493.

[28] R. Safavieh, G. Z. Zhou, D. Juncker, Lab Chip 2011, 11, 2618.

[29] M. Bayindir, F. Sorin, A. F. Abouraddy, J. Viens, S. D. Hart, J. D. Joannopoulos, Y. Fink, Nature 2004, 437, 826.

[30] A. F. Abouraddy, M. Bayindir, G. Benoit, S. D. Hart, K. Kuriki, N. Orf, O. Shapira, F. Sorin, B. Temelkuran, Y. Fink, Nature Mater. 2007, 6, 336

[31] M. Yaman, T. Khudiyev, E. Ozgur, M. Kanik, O. Aktas, E. O. Ozgur, H. Deniz, E. Korkut, M. Bayindir, Nature Mater. 2011, 10, 494.

[32] A. Yildirim, M. Vural, M. Yaman, M. Bayindir, Adv. Mater. 2011, 23, 1263.

[33] H. Lee, S. M. Dellatore, W. M. Miller, P. B. Messersmith, Science 2007, 318, 426.

[34] N. F. D. Vecchia, R. Avolio, M. Alfè, M. E. Errico, A. Napolitano, M. d'Ischia, Adv. Funct. Mater. 2013, 23, 1331.

[35] T. S. Sileika, D. G. Barrett, R. Zhang, K. H. A. Lau, P. B. Messersmith, Angew. Chem. Int. Ed. 2013, 52, 10766.

[36] R. A. Zangmeister, T. A. Morris, M. J. Tarlov, Langmuir 2013, 29, 8619.

[37] D. R. Dreyer, D. J. Miller, B. D. Freeman, D. R. Paul, C. W. Bielawski, Langmuir 2012, 28, 6428.

[38] J. Liebscher, R. Mrowczynski, H. A. Scheidt, C. Filip, N. D. Hadade, R. Turcu, A. Bende, S. Beck, Langmuir 2013, 29, 10539.

[39] P. B. Warren, Phys. Rev. E 2004, 69, 041601.

[40] J. A. Mann Jr., L. Romero, R. R. Rye, F. G. Yost, Phys. Rev. E 1995, 52, 3967.

[41] J. Tian, D. Kannangara, X. Lia, W. Shen, Lab Chip 2010, 10, 2258.

[42] Y. Wei, K. Li, S. Tong, Talanta 1996, 43, 1.

[43] K. H. Schosinsky, M. Vargas, A. L. Esquivel, M. A. Chavarria, Clin. Chem. 1987, 33, 223. 